\title{
Cost Efficiency Analysis of Space Debris Removal from Geostationary Orbit Using Service Spacecraft
}

\author{
V.E. Chebotarev \\ OOO Research and Production Center Small Satellites \\ 33 Schkolnaya Str., Building 25, Zheleznogorsk, the \\ Krasnoyarsk Territory, Russia, 662971
}

\author{
A.A. Vnukov \\ OOO Research and Production Center Small Satellites \\ 33 Schkolnaya Str., Building 25, Zheleznogorsk, the \\ Krasnoyarsk Territory, Russia, 662971 \\ E-mail: vnukovalx@ya.ru
}

\begin{abstract}
An economic efficiency model of spacecraft is developed. Besides, such economic efficiency indicators as profitability index and payback period of a project are designed. This model is modified to calculate the economic efficiency of a service spacecraft and as a result an overall index of project efficiency is developed on its basis. This index results in minimized interruptions when the system point is used in the geostationary orbit.
\end{abstract}

The analysis of conditions for the effective use of service spacecraft aimed at the removal of space debris from the geostationary orbit is carried out; the recommendations on its operating performance are made.

Keywords-spacecraft, space debris, geostationary orbit, system point, service spacecraft for space debris removal, cost efficiency.

\section{INTRODUCTION}

Today, space technologies have found widespread application in many areas of human activity in all regions of the Earth and near-Earth space, both air and outer space. It is difficult to imagine our modern life without long-distance and international satellite communication, centralized and direct broadcast satellite, satellite navigation, etc. This circumstance has defined the role of satellite space information support systems as a strategic means of ensuring national security and economic independence of any state $[1,2]$.

The growing demand for the application of space information support systems is largely fulfilled with the help of spacecraft (SC) placed in the geostationary orbit (GSO). As the GSO is very attractive for the international community, the geostationary spacecraft standpoints have been distributed between states and the requirements for the accuracy of the spacecraft holding at these points $\left( \pm 0.05^{\circ}\right.$ in longitude and inclination) have been determined. Today, the region of geosynchronous orbit is saturated with the operating spacecraft, as well as with those which service life has run out. Therefore, the international community (the Inter-Agency Space Debris Coordination Committee, IADC) has defined the protected areas of near-Earth space (Fig. 1) and the recommendations for the removal of non-operating $\mathrm{SC}$ at a sufficient distance from working GSO has been developed in order to avoid their interference with the operating spacecraft [1].

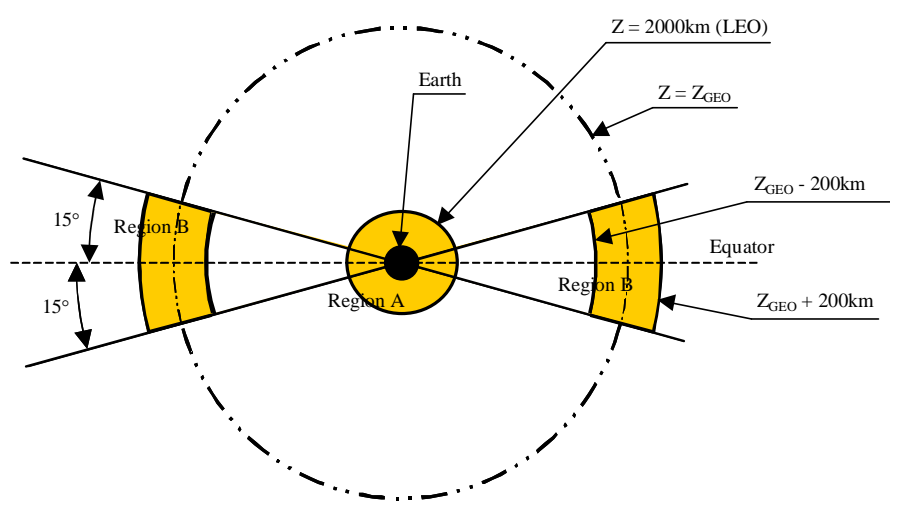

Fig. 1. Protected areas of near-Earth space

The altitude (above GSO) of the orbit of space debris disposal is defined in the document IADC Space Debris Mitigation Guidelines and by the national standards developed on the basis of this document following the formula [3, 4]:

$$
H_{O D}=235+1000 \cdot \frac{C_{R} A}{m},
$$

where, $\mathrm{H}_{O D}$ is the orbit altitude of space debris disposal above GSO $(\mathrm{km}), \mathrm{C}_{R}$ is the pressure coefficient of solar radiation, $A$ is the area of midsection of the spacecraft, $m$ is the dry mass of spacecraft.

However, the failure statistics shows that there are many cases when the spacecraft is out of control, i.e. its deorbiting is not possible. In this connection, the studies on the choice of means of active transfer of non-operating spacecraft into the disposal orbit, for example, with the help of a service spacecraft (SSC), as well as the development of cost effectiveness model of SSC application become relevant.

\section{ECONOMIC MODEL OF DESIGN AND OPERATION OF COMMUNICATION SPACECRAFT}

The lifecycle of a spacecraft (SC) is defined by the presence of the following stages [1]:

$$
\begin{aligned}
& \text { - R\&D works (R\&D); } \\
& \text { - operation } t_{O} \text { within the guaranteed life } t_{G L}\left(t_{O} \leq t_{G L}\right) \text {; } \\
& \text { - decommissioning. }
\end{aligned}
$$

The R\&D stage covers the period of time from making the decision on the beginning of works (final terms of reference, contract signing) until the end use, after putting the spacecraft into the orbit at the operational point with its presentation to 
the Customer on the basis of positive preliminary trials and is associated with large nonrecurring costs. At the operation stage, due to the end use (information support), there is an income, the total value of which (profit from the operation of the spacecraft received by the Customer) must exceed the $\mathrm{R} \& \mathrm{D}$ cost of at the end of the period $t_{G L}$.

The baseline costs for R\&D include the costs of developing and manufacturing the satellite, including its components, as well as preparation and launching the spacecraft into a working orbit.

The overall model of current net income from the project (SC) by analogy with the investment project is determined by the equation [5-7]

$$
C_{\text {c.n.i. }}=\left(C_{\text {s.i. }}-C_{\text {u.c. }}\right) t_{O}
$$

where, $C_{s . i .}, C_{u . c .}$ are the specific incomes and unit costs; $C_{R \& D . b}$ are the baseline costs for R\&D.

To a first approximation, the summands in the equation (2) are regarded independent of the guaranteed service life $\left(t_{G L}\right)$.

The functional dependence of the current net income on time is alternating: at the initial stage of satellite design it is negative, however, as the income increases it eventually becomes positive and at the end of operation period $\left(t_{O}=t_{G L}\right)$ it turns to the total net income

$$
C_{\text {s.n.i. }}=\left(C_{\text {s.i. }}-C_{\text {u.c. }}\right) t_{O}-C_{R \& D . b .}
$$

Basing on the overall model of current net income from the project (2), one can determine the payback period of the baseline costs for $\mathrm{R} \& \mathrm{D}$, assuming $C_{\text {c.n.i. }}=0$.

$$
t_{p p b s}=\frac{C_{R \& D . b .}}{C_{\text {s.i. }}-C_{u . c .}}
$$

Applying the expression for payback period of baseline costs $t_{p p b s}$ (4) to the equation of total net income from the operation period of the satellite (3) the following formula is obtained

$$
C_{\text {s.n.i. }}=C_{R \& D . b .}\left(\frac{t_{G L}}{t_{p p b s}}-1\right) \quad, t_{p p b s<} t_{G L}
$$

Basing on the formula (5) one may define the expression for calculating the relative dimensionless index of cost efficiency of the project, so-called the project profitability index [5-7]:

$$
K_{\text {i.p. }}=\frac{C_{\text {S.n.i. }}}{C_{R \& D . b .}}=\frac{t_{G L}}{t_{p p b s}}-1
$$

Following the condition $K_{\text {i.p. }}>1$ (guaranteed return of costs for R\&D) and basing on the formula (6) the upper limit of payback period of baseline costs $t_{p p b s} \leq 0,5 t_{G L}$ can be identified.

\section{ECONOMIC MODEL OF DESIGN AND OPERATION OF SERVICE SPACECRAFT}

The main goal for service spacecraft is the information support spacecraft in the GSO the control of which is violated as a result of any natural (spacecraft damaged by the meteoroid flow or the impact of high-power electromagnetic radiation pulse) or man-made anomalies (failure of one or several spacecraft systems as a result of a violation of the production or operation technology outside the design points).
The lifecycle of a service spacecraft is similar to the lifecycle of a communication spacecraft and is characterized by the presence of the following stages [1]:

- $R \& D$ works $(R \& D)$;

- operation $t_{O}$ within the guaranteed life $t_{G L}\left(t_{O} \leq t_{G L}\right)$;

- decommissioning.

The R\&D stage covers the period of time from making the decision on the beginning of works (final terms of reference, contract signing) until the end use of a spacecraft and after a service spacecraft orbiting $300 \mathrm{~km}$ higher compared to GSO altitude.

The operation of service spacecraft can be described as a multiple approach to space debris objects (SDO) and their deorbiting from the working orbit (GSO) to the disposal orbit. The maximum number of objects transported by one service spacecraft for the period of its operation $t_{G L}$ is determined by the equation

$$
n_{T O}=\frac{t_{G L}}{t_{d u r}} \leq n_{N S}, \quad n_{N S}=\frac{2 \pi}{\Delta \lambda},
$$

where, $t_{d u r}$ is the SC deorbiting duration from the working zone of GSO to the disposal orbit; $n_{T O}$ is the number of transported objects; $n_{N S}$ is the overall number of standpoints in the GSO; $\Delta \lambda$ is the longitude range of the point of standing (accuracy of geostationary spacecraft holding at the point of standing).

If a spacecraft fails, it stays at the point of standing for some period of time and then it begins to drift along the GSO. First of all, the negative effect of faulty spacecraft is the absence of ability to replace the spacecraft with a new one or working spacecraft (if an operator has it in the orbit or it is in the state of initial readiness on the Earth), and secondly, there is a risk of collision with another spacecraft in the GSO. Thus, the losses caused by the accidental spacecraft control loss in the GSO can be divided into three categories:

- Costs associated with the manufacturing (in the absence of a backup spacecraft) and launching a satellite into the orbit to replace an emergency spacecraft;

- Losses from the inability to operate the working point in the GSO;

- Decrease in profitability of the efficient spacecraft in other work points in the GSO due to the reduction in time of their active functioning caused by the need for additional maneuvering.

The first category of losses may be absent, if the operator of the communication satellite does not provide for the replacement of the emergency spacecraft, or if the failed device is replaced by another operator's spacecraft by transferring the operating spacecraft to the working point of the emergency spacecraft.

The second category of losses is associated either with the loss of the connection fees of the customers who cannot be provided with a service or with the need to rent a work point 
in the GSO (unless the operator is a state-owned company with an internationally fixed list of work points owned).

The third category of losses is a result of additional consumption of the working body of active spacecraft which are forced to make maneuvers to avoid collisions with SDO. Since a sufficient stock of the working body is the determining factor for extending the operation of a spacecraft beyond the warranty period, an additional maneuvering reduces the potential revenue of the satellite communications operator, which can be attributed to potential losses. In the event that any additional maneuvering in the future will lead to a shortage of the working body of a spacecraft correction system to be held in the GSO working point during the entire lifetime, the need for such maneuvering will be a direct loss for the satellite operator.

The income from service spacecraft operation would be the reduction of prohibition time of $t_{\Pi}$ for the application of standing point in the GSO for the intended purpose (lack of information support):

$$
t_{P}=\begin{aligned}
& t_{\text {break }}-\text { no } S S C \\
& t_{d u r}-\text { yes SSC }
\end{aligned}
$$

where, $t_{\text {break }}$ is the maximum break time during the replacement of failed spacecraft.

It can be seen from the expression (8) that the time of the ban on the use of the standing point corresponds to the time necessary for launching the backup spacecraft into the orbit (and, if necessary, the production of this backup spacecraft) or the time spent by the service spacecraft for approaching the failed spacecraft and displacing it to safety distance.

It is believed that with the reduction in the time of the ban on the use of the standing point, we obtain additional income from the target use of a spacecraft at this point:

$$
C_{a d d}=\left(C_{\text {s.i. }}-C_{u . c .}\right)\left(t_{\text {break }}-t_{d u r}\right)=C_{R \& D . S C} \frac{t_{\text {break }}-t_{d u r}}{t_{G L}}(9)
$$

As a result, the total net income from the application of a service spacecraft can be presented as follows

$$
C_{S S C}=C_{\text {add }} n_{T O} K_{\text {load }}-C_{R \& D . S S C}
$$

where, $K_{\text {load }}$ is the load factor of a service spacecraft aimed at servicing the GSO.

To increase the income from the operation of a service spacecraft, it is necessary to ensure that the load factor is as high as possible, that is, a new target for displacement into the disposal orbit must be known before the end of the previous displacement of a target.

Basing on the formula (10) one may define the expression for calculating the relative dimensionless indicator of the economic efficiency of the created project, so-called the profitability index of the project for service spacecraft development:

$$
K_{i d}=\frac{C_{S S C}}{C_{R \& D . S S C}}=n_{T O} K_{\text {load }} \frac{t_{\text {break }}-t_{\text {dur }}}{t_{G L}} \frac{C_{R \& D . S C}}{C_{R \& D . S S C}}-1
$$

It can be seen from the expression (11) that the profitability index of the project for the development of a service spacecraft, in contrast to the profitability index for the development of a communications satellite, depends not only on the time of operation of a service spacecraft, but also on the costs of communications satellite manufacturing.

\section{ANALYSIS OF DEVELOPED MODELS}

Basing on the condition $K_{i d} \geq 1$ (guaranteed return of $\mathrm{R} \& \mathrm{D}$ costs) one may find the upper limit of the payoff period of baseline costs using the following formula (11)

$$
K_{i d . s S C} \geq 1, \frac{C_{R \& D . S C}}{C_{R \& D . S S C}} \leq 0,5 n_{T O} K_{\text {load }} \frac{t_{\text {break }}-t_{d u r}}{t_{G L}} \frac{t_{G L}}{t_{p p b s}} \text {. }
$$

If to assume that $t_{o . \sigma} \leq 0,5 t_{\Gamma C}$ and to apply it to formula (12), we get

$$
\frac{C_{R \& D . S C}}{C_{R \& D . S S C}} \leq\left(\frac{t_{\text {break }}}{t_{\text {dur }}}-1\right) K_{\text {load }} .
$$

It follows from the inequality (13) that if $t_{\text {break }}<t_{\text {dur }}$, that is, when the approaching time of a service spacecraft and the space debris objects and its displacement to a safe distance is greater than the time spent by the operator for commissioning a new spacecraft, the ratio $\frac{C_{R \& D . S C}}{C_{R \& D . S S C}}$ becomes less than zero and the application of a service spacecraft is inexpedient. If the time of the interruption is equal to the time for displacement, both ways aimed to restore the operation of the working point are equivalent. Thus, to reduce the losses of satellite operators, a service spacecraft is advisable for the application only in the case when the displacement time of an emergency spacecraft to a safe distance from the working point is less than the commissioning time of a new spacecraft at the same point.

In the case when R\&D cost for the development of a service spacecraft is a part of $R \& D$ costs for the development of a spacecraft, there is a restriction on the ratio of time characteristics at different values of the load

$$
\delta C_{R \& D}=\frac{C_{R \& D . S S C}}{C_{R \& D . S S C}} \frac{t_{\text {break }}}{t_{\text {dur }}} \leq 1+\frac{\delta C_{R \& D}}{K_{\text {load }}}
$$

The obtained system of equations and inequalities makes it possible to determine the area of effective application of a service spacecraft and to determine a number of requirements to its technical specifications based on limitations on $R \& D$ cost for its development.

\section{CONCLUSION}

1. The goals and tasks of removing space debris from the geostationary orbit have been determined; technical features of the location of an emergency spacecraft in the disposal orbit have been considered.

2. Cost efficiency model of communication spacecraft has been developed, which makes it possible to estimate the payback period of a project based on the known costs for R\&D and specific economic indicators of spacecraft operation in the orbit. Based on this model, an estimation of the project profitability index was developed, which depends only on the time of operation of spacecraft for its intended purpose.

3. Cost efficiency model of service spacecraft has been developed. Besides, an overall index of project efficiency has 
been designed and generalized indicator of project efficiency, which ensures the minimization of interruptions in the application of a system point in the geostationary orbit, has been developed on its basis. Losses from the failure of a communication spacecraft in the GSO are divided into three categories: costs associated with the replacement of the emergency spacecraft by workers; losses from inability to operate the working point; decrease in profitability of the efficient spacecraft at other points in the geostationary orbit (GSO). A direct dependence of the income from the operation of service spacecraft with the load factor has been revealed.

4. The analysis of conditions for the effective application of a service spacecraft has been carried out. The futility boundary for service spacecraft aimed at the reduction of losses of communication operators caused by failed spacecraft is indicated: the transfer time of the emergency spacecraft to the disposal orbit must be less than the time required for the operator to replenish the orbital grouping.

\section{Acknowledgements}

The work was carried out in the framework of applied scientific research on the topic Development of the Concept of a Service Spacecraft for Cleaning Geostationary Orbit (GSO) from Man-Made Space Debris with the financial support of the Ministry of Education and Science of the Russian Federation. The unique identifier of the agreement is RFMEFI57617X0093.

\section{References}

[1] V.E. Chebotarev, V.E. Kosenko, Fundamentals of the design of information support spacecraft: Textbook, Siberian State Aerospace University, Krasnoyarsk, 2011.

[2] V.V. Makarov, Telecommunications of Russia: state, trends and ways of development: Monograph, M.: IRAS, 2007.

[3] IADC Space Debris Mitigation Guidelines, Inter-agency Space Debris Coordination Committee site. IADC-02-01, 2007. Retrieved from: http://www.iadc-online.org/Documents/IADC-200201,\%20IADC\%Space\%20Debris\%20Guidelines,\%20Revision\%201.pdf

[4] GOST R 52925-2008, Products of space technology. General requirements for space facilities to limit man-made pollution of nearEarth space, Intr. 2009-01-01. M.

[5] V.I. Titov, The economy of enterprise: Textbook, M; Eksmo, 2008.

[6] Yu.P. Lukashin, Financial Mathematics: Educational and methodological complex, M: publishing house Center of the EAOI, 2008.

[7] V.E. Chebotarev, V.V. Popov "Index rating system of cost efficiency of communication satellites," Bulletin of Siberian State University of Agriculture, Vol. 6 (52), pp. 60-64, 2013. 